\title{
Prognostic Value of the Immunological Subtypes of Adolescent and Adult T-Cell Lymphoblastic Lymphoma; an Ultra-High-Risk Pro-T/CD2(-) Subtype
}

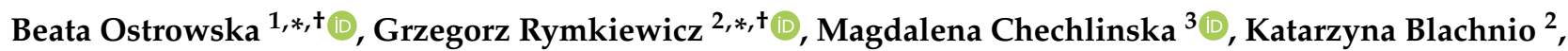 \\ Katarzyna Domanska-Czyz ${ }^{1}$, Zbigniew Bystydzienski ${ }^{2}$, Joanna Romejko-Jarosinska ${ }^{1}$, Anita Borysiuk ${ }^{2}$, \\ Sebastian Rybski ${ }^{4}$, Wojciech Michalski ${ }^{4}$ and Jan Walewski ${ }^{1}$ (i)
}

check for updates

Citation: Ostrowska, B.; Rymkiewicz, G.; Chechlinska, M.; Blachnio, K.;

Domanska-Czyz, K.; Bystydzienski, Z.; Romejko-Jarosinska, J.; Borysiuk, A.; Rybski, S.; Michalski, W.; et al. Prognostic Value of the Immunological Subtypes of Adolescent and Adult T-Cell Lymphoblastic Lymphoma; an Ultra-High-Risk Pro-T/CD2(-) Subtype. Cancers 2021, 13, 1911. https://doi.org/10.3390/ cancers13081911

Academic Editor: Armand Bensussan

Received: 9 March 2021

Accepted: 12 April 2021

Published: 15 April 2021

Publisher's Note: MDPI stays neutral with regard to jurisdictional claims in published maps and institutional affiliations.

Copyright: (c) 2021 by the authors. Licensee MDPI, Basel, Switzerland. This article is an open access article distributed under the terms and conditions of the Creative Commons Attribution (CC BY) license (https:// creativecommons.org/licenses/by/ $4.0 /)$.
1 Department of Lymphoid Malignancies, Maria Sklodowska-Curie National Research Institute of Oncology, 02-781 Warsaw, Poland; katarzyna.domanska-czyz@pib-nio.pl (K.D.-C.); joanna.romejko-jarosinska@pib-nio.pl (J.R.-J.); jan.walewski@pib-nio.pl (J.W.)

2 Flow Cytometry Laboratory, Department of Pathology and Laboratory Diagnostics, Maria Sklodowska-Curie National Research Institute of Oncology, 02-781 Warsaw, Poland; katarzyna.blachnio@pib-nio.pl (K.B.); Zbigniew.Bystydzienski@pib-nio.pl (Z.B.); anita.borysiuk@pib-nio.pl (A.B.)

3 Department of Cancer Biology, Maria Sklodowska-Curie National Research Institute of Oncology, 02-781 Warsaw, Poland; magdalena.chechlinska@pib-nio.pl

4 Department, of Mathematical Oncology, Maria Sklodowska-Curie National Research Institute of Oncology, 02-781 Warsaw, Poland; sebastian.rybski@pib-nio.pl (S.R.); wojciech.michalski@pib-nio.pl (W.M.)

* Correspondence: beata.ostrowska@pib-nio.pl (B.O.); grzegorz.rymkiewicz@pib-nio.pl (G.R.)

+ These authors contributed equally to this work.

Simple Summary: T-cell lymphoblastic lymphoma (T-LBL) is extremely rare and aggressive with no practical risk model defined. Considering the controversies over the prognostic value of T-LBL immunological subtypes, we re-evaluated 49 subsequent adult T-LBL patients treated according to the German Multicenter Study Group for Adult Acute Lymphoblastic Leukemia protocols, with $85.7 \%$ with complete remissions. To the best of our knowledge, this is the largest study of T-LBL diagnosed by flow-cytometry of the material obtained by fine-needle aspiration biopsy. We show that (1) CD2 status and age are powerful independent prognostic factors influencing overall survival and the risk of treatment failure; (2) the early/pro-T/CD2(-) subtype is associated with extremely poor outcomes; and (3) poor outcomes in ETP vs. non-ETP are strikingly consistent with the pro-T CD2(-) subtype. The lack of CD2 expression in T-LBL emerges as a new marker of an ultra-high-risk of treatment failure. We show here that ETP is a non-uniform entity, where the outcome depends on the CD2 status.

Abstract: (1) Background: T-cell lymphoblastic lymphoma (T-LBL) is extremely rare and highly aggressive, with no practical risk model defined yet. The prognostic value of T-LBL immunological subtypes is still a matter of controversy. (2) Methods: We re-evaluated 49 subsequent adult T-LBL patients treated according to the German Multicenter Study Group for Adult Acute Lymphoblastic Leukemia (GMALL) protocols, $05 / 93(n=20)$ and T-LBL 1/2004 $(n=29), 85.7 \%$ of which achieved complete remission (CR). (3) Results: The 5/10-year overall survival (OS) and event-free survival (EFS) were $62 \% / 59 \%$ and $48 \% / 43 \%$, respectively. In $96 \%$ of patients, flow cytometry analyses defining the WHO 2008 immunophenotypes were available. Cortical, early/pro-T/CD2(-), early/pre-T/CD2(+), and mature subtypes were identified in 59.5\%,19\%, 15\%, and $6.5 \%$ of patients, respectively. Overall, $20 \%$ of patients had the early T-cell precursor (ETP)-LBL immunophenotype, as proposed by the WHO 2017 classification. For the early/pro-T/CD2(-) subtype, the five-year OS and EFS were $13 \%$ and $13 \%$, while for all the other, non-pro-T subtypes, they were $69 \%$ and $67 \%$. By multivariate analysis, only CD2(-) status and age $>35$ years emerged as strong, independent factors influencing OS and EFS, while the risk of CR failure was influenced by age only ( $>35$ years). (4) Conclusions: ETP was non-significant for OS, unless an ultra-high-risk pro-T/CD2(-) subtype was concerned. 
Keywords: CD2; T-LBL; T-ALL/LBL; lymphoblastic lymphoma; flow cytometry

\section{Introduction}

T-cell lymphoblastic lymphoma (T-LBL) is an aggressive, very rare malignancy from precursor thymic $\mathrm{T}$ cells transformed at different stages of differentiation. According to the definition given by the WHO, T-LBL is classified together with acute lymphoblastic leukemia (T-ALL) as T-ALL/LBL $[1,2]$. The current standard of treatment for adult LBL patients is the ALL-like intensive therapy [3,4]. Despite an overall complete remission (CR) rate of 76-93\%, one-third of patients relapse, and the 5-year overall survival (OS) rate for T-LBL is $51-69 \%$ in prospective studies [5-9] and $42-48 \%$ according to national epidemiology databases [10,11]. In ALL, the risk factors of treatment failure have been quite well recognized and allow, among others, to qualify patients for allogeneic stem cell transplantation (allo-SCT). Contrary to this, in LBL, no practical risk model has yet been defined. The main role in an ALL risk model is attributed to the level of minimal residual disease (MRD), but for LBL patients, MRD tracking is limited [7]. Most oncologists and hematologists regard immunological subtypes as important prognostic factors, with cortical CD1a(+) T-ALL/LBL having a considerably better prognosis than early-T or mature T-ALL/LBL [12]. Most published data distinguish immunological subtypes considering the status of CD1a and surface (s)CD3 only, but not the CD2 status, and as a result the pro-T/CD2(-) and pre-T/CD2(+) subtypes fall into a single, early subtype (CD1a-/sCD3-) [12-14]. In contrast to T-ALL, few data are available on the incidence and prognostic value of immunologic subtypes of adolescent/adult T-LBL [9,15]. The prognostic impact of immunological subtypes may be especially significant in T-LBL, where most often there is no possibility to follow the MRD. Recently, a subtype of T-ALL/LBL derived from thymic cells at the early T-cell precursor (ETP) differentiation stage has been recognized as having an extremely poor prognosis [2,13,16-19]. Considering the uncertain prognostic value of T-LBL immunological subtypes in the context of the 2008 and 2017 WHO classifications [1,2], we reevaluated 49 consecutive adolescent/adult patients. We analyzed their outcomes along with the expression of differentiation markers to examine the influence of immunological subtypes on prognosis. All T-LBL patients followed frontline regimens according to the German Multicenter Study Group for Adult ALL (GMALL) protocols [5,9]. Flow cytometry (FCM) immunophenotyping of the cellular suspension, obtained by fine needle aspiration biopsy (FNAB) or by ultrasound-guided or computed tomography (CT)-guided-FNAB from the involved lymph nodes or mediastinal tumors, was performed.

\section{Materials and Methods}

Forty-nine adult patients with T-LBL treated at the Maria Sklodowska-Curie National Research Institute of Oncology between 2000 and 2018 were enrolled in the analyses. All patients met the diagnostic criteria of T-LBL, based on the identification of a neoplastic proliferation of blasts with cytoplasmic CD3 (cCD3) expression and usually also a nuclear expression of terminal deoxynucleotidyl transferase (TdT). A cutoff of $<25 \%$ bone marrow (BM) blasts was used to define LBL [1,2]. The diagnosis of T-LBL was made by an expert hematopathologist, routinely performing FNAB/FCM, assessing MRD, and undertaking histopathology (HP) and immunohistochemical (IHC) examinations. In 47 patients, the immunological subtype was defined according to the 2008 WHO classification as pro- $\mathrm{T}(\mathrm{cCD} 3+/ \mathrm{sCD} 3-/ \mathrm{CD} 2-/ \mathrm{CD} 7+/ \mathrm{CD} 1 \mathrm{a}-/ \mathrm{CD} 4-/ \mathrm{CD} 8-/ \mathrm{CD} 34+/-)$, pre-T (cCD3+/sCD3-/CD2+/ CD7+/CD1a-/CD4-/CD8-/CD34+/-), cortical T (cCD3+/ sCD3-/CD2+/CD7+/CD1a+/ CD4+/CD8+/CD34-), or medullary T (cCD3+/sCD3+ $/ \mathrm{CD} 2+/ \mathrm{CD} 7+/ \mathrm{CD} 1 \mathrm{a}-/ \mathrm{CD} 34-/ \mathrm{CD} 4+$ or $\mathrm{CD} 8+)$ [1]. The ETP subtype was defined according to the $2017 \mathrm{WHO}$ classification and was distinguished by the following immunophenotype: $\mathrm{CD} 1 \mathrm{a}(-) / \mathrm{CD} 8(-)$ and $\mathrm{CD} 5(-)$ or $\mathrm{CD} 5(+)^{\text {weaker }}$ with stem cell or myeloid marker 
expression [2,16]. The immunophenotype was determined by the FCM of cellular suspensions obtained before treatment from the involved lymph nodes $(n=21)$, mediastinal mass $(n=12)$, or nasopharyngeal/perimandibular infiltration $(n=2)$ by FNAB, and also from $\mathrm{BM}(n=2)$, peripheral blood $(\mathrm{PB})(n=1)$, and pleural fluid $(n=7)$. Four to ten separate needle passes within a lymph node or tumor (three or four passes within mediastinal mass) provided adequate cellular material. FNABs were carried out with needles no. 21-23G and syringes rinsed in physiological saline (PBS) with $\mathrm{K}_{2}$ EDTA (ethylenediaminetetraacetic acid). A minimal panel of anti-human monoclonal antibodies ( $\mathrm{mAb}$ ) used for the FCM analysis to evaluate T-ALL/LBLs and ETP included antibodies against $\mathrm{CD}(1 \mathrm{a} / 2 / \mathrm{c} 3 / \mathrm{s} 3 / 4 / 5 / 7 / 8 / 13 / 15 / 33 / 34 / \mathrm{c} 79 \alpha), \mathrm{MPO} / \mathrm{TdT}$, and HLADR (Table S1A). Additional mAbs used for extended FCM diagnostics are included in the Supplementary Methods and listed in Table S1B. Because of immunophenotype diversity related to the ontogenetic maturity of T-LBL, within one half-hour before the full panel was tested, we assessed a basic lymphoma panel (an initial test) in order to set the ultimate individual panel for each patient. FNAB samples were prepared by a standard technique of 3- or 4-color immunophenotyping as previously described [20]. For T-LBL diagnosis, cells obtained by FNAB of the lymph nodes/tumors were immunophenotyped as previously described [20]. For CD2 expression, PE-conjugated CD2 antibody clone S5.2, Becton Dickinson Biosciences, $\mathrm{BD}$, was used; all mAbs are specified in Supplementary Methods. Antigen (Ag) expression was quantified by FACScan (until 2000y), FACSCalibur (until 2007y), and FACSCanto II (to this day) cytometers (BD, San Jose, CA, USA), and categorized into three groups according to the percentages of positive cells: " $(-)$ ", not expressed, if detected in $<20 \%$ of neoplastic cells; " $(+/-)$ ", if expressed in $\geq 20 \%$ and $<100 \%$ of neoplastic cells; " $(+)$ ", if expressed in $100 \%$ of lymphoma cells. Expression was quantified as $(+)^{\text {weaker }}$ or $(+)^{\text {higher }}$ than that on control $\mathrm{T}$ lymphocytes, as previously described $[20,21]$. Cells obtained by FNAB were also stained with a rapid hematoxylin and eosin stain, and smears performed from $\mathrm{BM} / \mathrm{PB}$ were stained with the May-Grünwald-Giemsa for morphological evaluation. In 47 patients diagnosed by FNAB/FCM, 43 patients were diagnosed also by HP/IHC of trephine bone marrow and surgical biopsies. On top of that, two patients had HP only with a limited IHC panel, without FCM. Between 2000 and 2006, immunohistochemical diagnosis of T-LBL was based on the expression of a limited number of antigens, i.e., CD3, CD34, TdT, CD20, Ki-67, and MPO. In 2007, the IHC panel was supplemented with CD1a, CD2, CD4, CD5, CD7, and CD8, necessary to assess the T-LBL immunological subtypes. In 10 patients, CD2 was assessed by both immunohistochemical staining and FCM (four CD2-negative and six CD2-positive). An IHC reaction for CD2 was considered positive if any of the T-LBL cells showed staining, against control normal $\mathrm{T}$ lymphocytes which are always CD2(+) ${ }^{\text {higher }}$. The IHC results were consistent with FCM in all cases. The diagnostic material taken by FNAB, if sufficient, was also sent for cytogenetics, as previously described [20]. Patients were treated according to GMALL 05/93 (years 2000-2004) and GMALL T-LBL 1/2004 (years 2005-2018) protocols (Table S2) [5,9]. CR was defined as a resolution of extramedullary disease, normal BM status ( $\leq 5 \%$ blasts), neutrophil count $\geq 1.0 \mathrm{G} / \mathrm{L}$, and platelet count $\geq 100 \mathrm{G} / \mathrm{L}$. The mediastinal mass response was assessed by $\mathrm{CT}$ imaging. It was considered residual if there was a decrease in dimension of over $75 \%$ that was stable in two subsequent CT examinations. Since September 2010, positron emission tomography/computed tomography has been routinely used to confirm CR after second induction (seventh week of treatment).

\section{Statistical Analysis}

OS was calculated from the date of treatment initiation to death or to the date of the last follow-up in surviving patients. Event-free survival (EFS) was calculated from the beginning of treatment until an event (relapse, treatment failure, death during induction, or death during complete remission). Patients with stem cell transplantation (SCT) were censored at the time of transplantation. Survival curves were calculated by the KaplanMeier method and compared with the log-rank test. Differences of frequency were analyzed 
with the Chi-squared or Fisher exact test. Univariate and multivariate analyses were performed to identify prognostic factors (age, white blood cell (WBC) count, hemoglobin, platelet count, central nervous system (CNS) involvement at diagnosis, BM involvement at diagnosis, GMALL 05/93 vs. GMALL 2004 treatment, WHO classification (early/pro-T, early / pre-T, cortical, mature) and ETP vs. non-ETP, expression of $\mathrm{CD}(1 \mathrm{a} / 2 / \mathrm{s} 3 / 4 / 8 / 5 / 7)$ vs. non-expression, and the number of pan-T Ags expressed (0-3 vs. 4-7). The Cox Proportional Hazard Model (PHM) was used for factor analysis of the main endpoints, and the logistic regression model was applied for secondary endpoint analysis. A significance level of $p<0.05$ was set for all statistical tests. The IBM SPSS 23 package was used for statistical analysis.

\section{Results}

Between 2000 and 2018, 49 adult patients with T-LBL were treated according to the GMALL 05/93 $(n=20)$ and GMALL T-LBL 1/2004 $(n=29)$ protocols. The median age of patients at diagnosis was 28 years (range 16-56), with 35 (71.4\%) younger than 35 years, and the majority of patients were males $(75.5 \%)$. The most typical location of the disease was bulky mediastinal mass (92\%), with simultaneous pleural effusion in almost half of the patients $(47 \%)$ and pericardial effusion in one third $(35 \%)$. The mean WBC count was 9.9 G/L (range, 2.5-21.2), HGB $13.7 \mathrm{~g} / \mathrm{dl}$ (range, 9.1-17.7), and PLT $313 \mathrm{G} / \mathrm{L}$ (range, 151-788). Based on the HP assessment of trephine bone marrow biopsy specimens, bone marrow involvement was found in $22 \%(n=11)$ of patients. Classical trephine bone marrow biopsy and FCM evaluations were performed simultaneously in 15 patients. In nearly half of them $(7 / 15(46.6 \%))$, low BM involvement was evidenced by FCM, with no evidence of involvement in classical trephine bone marrow biopsy. Primary CNS involvement concerned only three patients $(6 \%)$. The baseline characteristics of the T-LBL patients are detailed in Table S3. Overall, $42(85.7 \%)$ of the patients achieved CR. The median follow-up was 155.6 months. The 5/10-year OS and EFS were $62 \% / 59 \%$ and $48 / 43 \%$, respectively. Forty-seven patients $(96 \%)$ had a comprehensive FCM analysis to define subtypes according to the $2008 \mathrm{WHO}$ criteria for T-ALL/LBL, based on the status of the pan-T Ag panel: CD1a, CD2, sCD3, CD4, CD5, CD7, and CD8. The most common T-LBL was of the cortical subtype (59.5\%), followed by the early subtype (34\%), subdivided by CD2 status to the early/pro-T/CD2(-) (19\%), and the early/pre-T/CD2(+) (15\%), while the least frequent was the mature subtype (6.5\%). In part of cases, we managed to set the karyotype. A complex karyotype was identified in 7/8 (88\%) CD2(-) cases and 10/18 (56\%) CD2(+).

The 5/10-year OS and EFS were strongly related to the 2008 WHO subtypes. For the cortical subtype, $5 / 10$-year OS and EFS were $75 \% / 69 \%$ and $75 \% / 68 \%$, while for the non-cortical subtypes they were $38 \% / 38 \%$ and $31 \% / 31 \%$, respectively $(p=0.023 / 0.002)$ (Figure 1).

For the early / pro-T subtype, the 5-year OS and EFS were 13\% and 13\%, while for all the other non-pro-T they were $69 \%$ and $67 \%$, respectively $(p=0.001 /<0.001)$ (Figure 2 ).

The five-year OS for patients with CD2, CD1a, and more than three of the pan-T Ag panel expressions was $73 \%, 78 \%$, and $76 \%$, compared to $26 \%, 34 \%$, and $28 \%$ for patients without $\mathrm{CD} 2$ and CD1a and no more than three of the pan-T Ag expressions, respectively $(p=0.001 /=0.002 /=0.001)$ (details in Table 1, Figures 3 and 4).

By univariate analysis, the following variables significantly influenced survival: age ( $\leq 35$ vs. $>35$ ); the number of pan-T Ags ( $4-7$ vs. $0-3$ present); and CD1a, CD2, sCD3, and CD8 present vs. absent. By multivariate analysis, only age $>35$ years (hazard ratio (HR), 5.39 ; $95 \%$ confidence interval $(\mathrm{CI}), 2.10-13.8 ; p<0.001)$ and the lack of CD2 expression (HR, 5.10; 95\% CI, 1.93-13.49; $p<0.001)$ were powerful independent prognostic factors, influencing OS. Similarly, age $>35$ years and CD2(-) status were the only significant factors for EFS ( $p=0.001$ and $p<0.001$, respectively), details in Table 2 . 


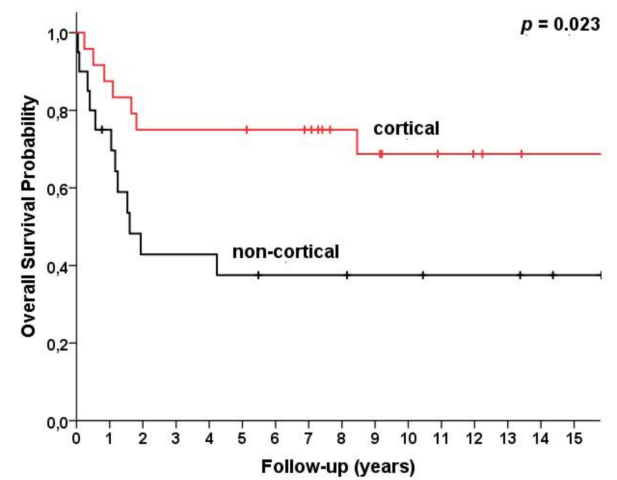

(a)

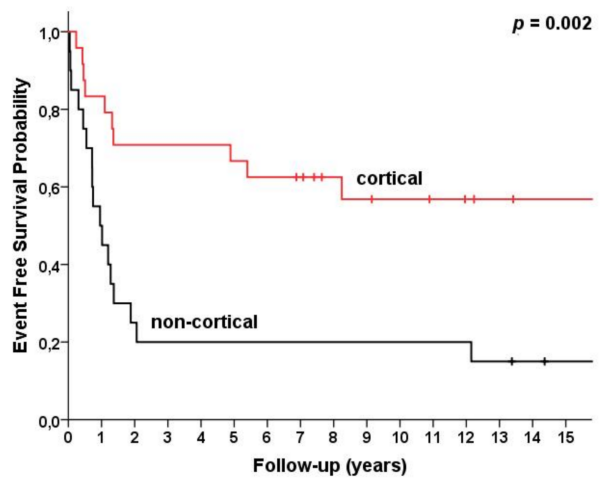

(b)

Figure 1. Survival for T-cell lymphoblastic lymphoma (T-LBL) $(n=47)$ patients categorized by immunological subtypes (WHO 2008) as cortical $(n=28)$ vs. non-cortical $(n=19)$ subtype. (a) Overall survival and (b) event-free survival.

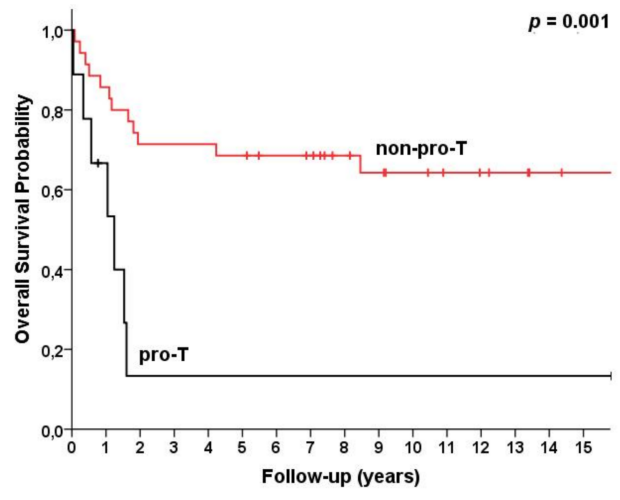

(a)

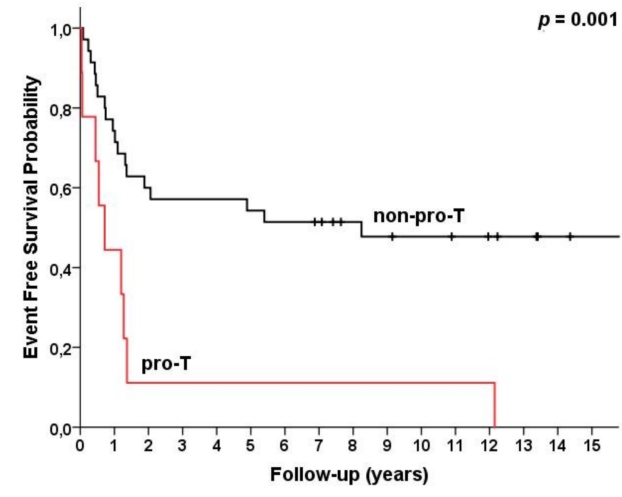

(b)

Figure 2. Survival for T-LBL $(n=47)$ patients categorized by immunological subtypes (WHO 2008) as early/pro-T ( $n=9)$ vs. non-pro-T $(n=38)$ subtype. (a) Overall survival and (b) event-free survival.

Table 1. Overall survival (OS) and event-free survival (EFS) according to age $(n=49)$, immunological subtypes and number of pan-T antigens $(n=47)$.

\begin{tabular}{|c|c|c|c|c|c|c|c|}
\hline \multirow{2}{*}{ Variables } & \multirow{2}{*}{$n$} & \multicolumn{2}{|c|}{ OS } & \multirow{2}{*}{$p$} & \multicolumn{2}{|c|}{ EFS } & \multirow{2}{*}{$p$} \\
\hline & & $\begin{array}{c}5 \text { Year }(\%) \\
(95 \% \text { CI) }\end{array}$ & $\begin{array}{c}10 \text { Year (\%) } \\
(95 \% \text { CI) }\end{array}$ & & $\begin{array}{c}5 \text { Year }(\%) \\
(95 \% \text { CI) }\end{array}$ & $\begin{array}{c}10 \text { Year (\%) } \\
(95 \% \text { CI) }\end{array}$ & \\
\hline & 49 & $62(48 ; 76)$ & $59(44 ; 74)$ & - & $48(33 ; 63)$ & $43(28 ; 58)$ & - \\
\hline Age $\leq 35$ & 35 & $76(62,91)$ & $76(62,91)$ & & $73(58,89)$ & $73(58,89)$ & \\
\hline Age $>35$ & 14 & $28(5,52)$ & $14(0,37)$ & $<0.001$ & $26(2,50)$ & $13(0,34)$ & $<0.001$ \\
\hline Cortical & 28 & $75(58,92)$ & $69(49,89)$ & & $75(58,92)$ & $68(48,88)$ & \\
\hline Non-cortical & 19 & $38(16,59)$ & $38(16,59)$ & 0.023 & $31(8,54)$ & $31(8,54)$ & 0.002 \\
\hline Pro-T & 9 & $13(0,38)$ & $13(0,25)$ & & $13(0,38)$ & $13(0,38)$ & \\
\hline Non-proT & 35 & $69(53,84)$ & $64(47,81)$ & 0.001 & $67(51,83)$ & $63(45,80)$ & $<0.001$ \\
\hline ETP & 9 & $33(3,64)$ & $33(3,64)$ & 0135 & $26(0,57)$ & $26(0,57)$ & \\
\hline Non-ETP & 38 & $66(50,82)$ & $61(45,78)$ & 0.135 & $63(47,80)$ & $59(41,76)$ & 0.095 \\
\hline CD1a(+) & 28 & $78(62,94)$ & $72(54,90)$ & & $76(59,93)$ & $69(49,89)$ & \\
\hline CD1a(-) & 19 & $34(12,56)$ & $34(12,56)$ & 0.002 & $28(6,51)$ & $28(6,51)$ & 0.001 \\
\hline $\mathrm{CD} 2(+)$ & 34 & $73(58,88)$ & $68(52,85)$ & & $71(55,87)$ & $66(48,84)$ & $<0001$ \\
\hline $\mathrm{CD} 2(-)$ & 13 & $26(1,51)$ & $26(1,51)$ & 0.001 & $19(0,43)$ & $19(0,43)$ & $<0.001$ \\
\hline 0-3 panT Ags expressed & 16 & $28(5,50)$ & $28(5,50)$ & 0.001 & $18(0,40)$ & $18(0,46)$ & $<0.001$ \\
\hline 4-7 panT Ags expressed & 30 & $76(61,92)$ & $71(54,89)$ & 0.001 & $74(57,72)$ & $68(49,87)$ & $<0.001$ \\
\hline
\end{tabular}




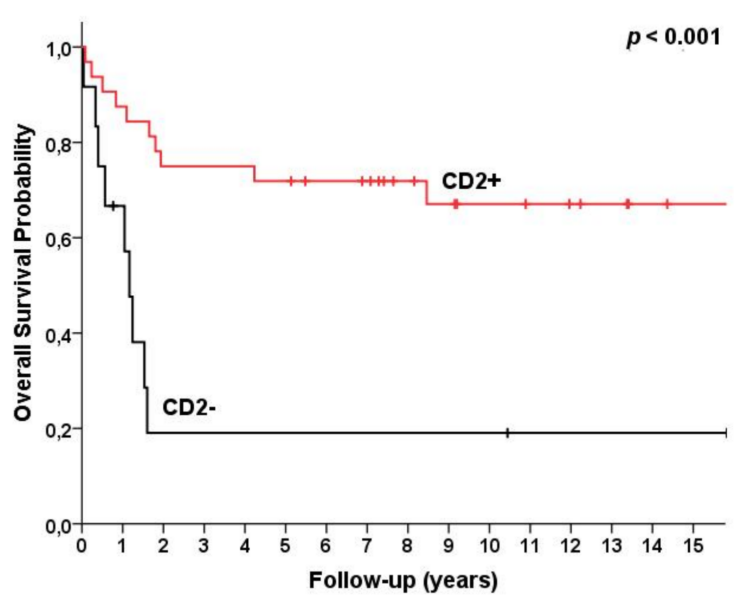

(a)

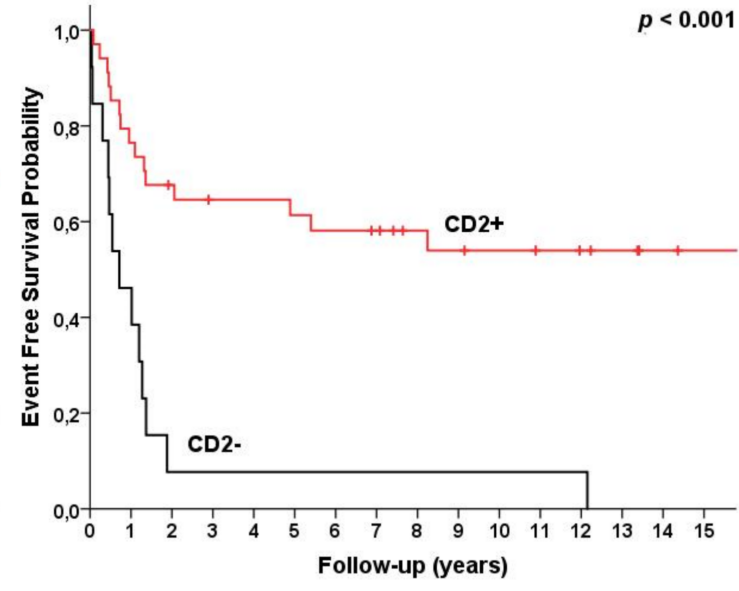

(b)

Figure 3. Survival for T-LBL $(n=47)$ patients categorized by CD2 status as CD2 present $(n=34)$ vs. CD2 absent $(n=13)$. (a) Overall survival and (b) event free survival.

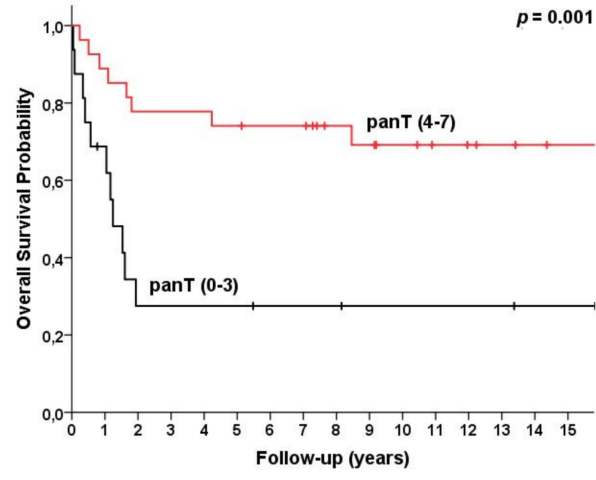

(a)

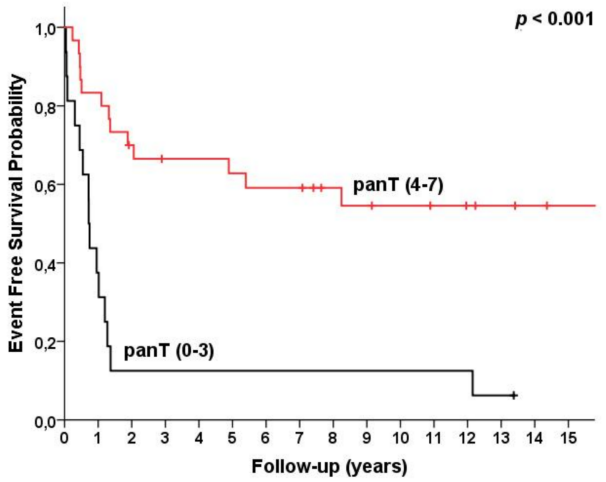

(b)

Figure 4. Survival for T-LBL $(n=46)$ patients categorized by number of pan-T Ags as pan-T 0-3 Ags present $(n=16)$ vs. $4-7$ Ags present $(n=30)$. (a) Overall survival and (b) event-free survival.

There was a significant correlation between CD2 expression and the expression of CD1a $(p<0.001), \operatorname{sCD} 3(p=0.002), \mathrm{CD} 4(p=0.002), \mathrm{CD} 8(p<0.001)$, and of more than three pan-T Ags $(p<0.001)$, the cortical subtype $(p<0.001)$, and subtypes other than the pro-T $(p<0.001)$. Overall, $27.6 \%(n=13)$ of patients were CD2 $(-)$, including $69 \%(n=9)$ of patients with the early/pro-T subtype (with 2-3 of pan-T Ags only), two patients with the medullary subtype, and two patients with an unusual CD2(-) cortical subtype. Nearly half $(n=6)$ of the CD2 $(-)$ patients met the criteria of the ETP subtype. The median age was 30 years (range, 18-57), with eight (61\%) younger than 35 years; male sex was dominant (92\%). Only 69\% $(n=9)$ of the CD2 $(-)$ patients achieved CR, compared with 91\% $(n=31)$ in the CD2 $(+)$ patients, and $77 \%(n=10)$ progressed, compared with only $20.5 \%(n=7)$ in the CD2(+) patients. Of the $13 \mathrm{CD} 2(-)$ patients, five $(38.5 \%)$ received SCT, among them two in the first-line treatment (CR1) and three in the second remission (CR2). All four CD2 $(-)$ patients who are alive $(30.7 \%)$ have undergone SCT: one in CCR1, now three years after allo-SCT, and the other three in CCR2 (one of those relapsed 11 years after CR1, was transplanted in CR2, and now it is his fifth year without evidence of disease; the other two are without evidence of disease 11 and 3 years after allo-SCT). Baseline characteristics and outcomes for CD2(-) vs. CD2(+) are detailed in Table 3 (47 patients diagnosed by FNAB/FCM) and Table S4 (49 patients, including two with CD2 expression detected by IHC). 
Table 2. Results of multivariate analysis for OS, EFS, and CR endpoints.

\begin{tabular}{ccccc}
\hline CPHM & Variables & HR & $\mathbf{9 5 \% C I}$ & $p$ \\
\hline \multirow{2}{*}{ OS } & Age $>35$ & 5.39 & $2.10-13.8$ & $<0.001$ \\
& CD2 negative & 5.10 & $1.93-13.49$ & 0.001 \\
\multirow{2}{*}{ EFS } & Age $>35$ & 3.77 & $1.72-8.27$ & 0.001 \\
& CD2 negative & 4.52 & $2.03-10.06$ & $<0.001$ \\
\hline \multirow{2}{*}{ LRM } & Variables & OR & $\mathbf{9 5 \% C I}$ & $p$ \\
\hline \multirow{2}{*}{ CR } & Age $>35$ & 0.039 & $0.004-0.413$ & 0.007 \\
& CD2 negative & 0.193 & $0.025-1.50$ & 0.116 \\
\hline
\end{tabular}

Abbreviations: CPHM-Cox Proportional Hazard Model, LRM-logistic regression model, HR—hazard ratio, $\mathrm{OR}$-odds ratio, CI—confidence interval, OS — overall survival, EFS— event-free survival, CR—complete remission.

Table 3. Baseline characteristics and outcomes for CD2(-) patients compared to CD2(+) patients diagnosed by fine needle aspiration biopsy (FNAB)/ flow cytometry (FCM).

\begin{tabular}{|c|c|c|c|}
\hline$n(\%)$ & $\begin{array}{c}\text { Total } \\
n=47(100 \%)\end{array}$ & $\begin{array}{c}\text { CD2(+) } \\
n=34(100 \%)\end{array}$ & $\begin{array}{c}\text { CD2(-) } \\
n=13(100 \%)\end{array}$ \\
\hline Gender, male & $36(76.5 \%)$ & $24(70.5 \%)$ & $12(92 \%)$ \\
\hline Age, median (range) & $28(18,58)$ & $26(18,58)$ & $30(18,57)$ \\
\hline$<35$ year & $33(70 \%)$ & $25(73.5 \%)$ & $8(61 \%)$ \\
\hline \multicolumn{4}{|l|}{ Treatment } \\
\hline GMALL 05/93 & $18(38 \%)$ & $13(38 \%)$ & $5(38.5 \%)$ \\
\hline GMALL 01/2004 & $29(62 \%)$ & $21(62 \%)$ & $8(61.5 \%)$ \\
\hline $\begin{array}{c}\text { Bone marrow } \\
\text { involvement }(<25 \%)\end{array}$ & $11(23 \%)$ & $7(20.5 \%)$ & $4(30.5 \%)$ \\
\hline \multirow[t]{2}{*}{ CNS involvement } & $3(6 \%)$ & $2(6 \%)$ & $1(7.5 \%)$ \\
\hline & median (range) & median (range) & median (range) \\
\hline WBC (G/L) & $9.2(2.5-21.2)$ & $8.6(2.5-21.2)$ & $10(3-18)$ \\
\hline HGB (g/dl) & $13.8(9.1-17.7)$ & $13.4(9.1-16.9)$ & $14(9.3-17.7)$ \\
\hline $\operatorname{PLT}(\mathrm{G} / \mathrm{L})$ & 317 (151-788) & $296(151-754)$ & 349 (198-788) \\
\hline \multicolumn{4}{|l|}{ Immunophenotype } \\
\hline Pro-T & $9(19 \%)$ & & $9(69 \%)$ \\
\hline Pre-T & $7(15 \%)$ & $07(20.5 \%)$ & 0 \\
\hline Cortical & $28(59.5 \%)$ & $26(76.5 \%)$ & $2(15.5 \%)$ \\
\hline mature & $3(6.5)$ & $1(3 \%)$ & $2(15.5 \%)$ \\
\hline $\mathrm{ETP} *$ & $9(20 \%)$ & $3(9 \%)$ & $6(46 \%)$ \\
\hline $\mathrm{CR}$ & $40(85 \%)$ & $31(91 \%)$ & $9(69 \%)$ \\
\hline progression & $17(36 \%)$ & $7(20.5 \%)$ & $10(77 \%)$ \\
\hline alive & $28(59.5 \%)$ & $24(70.5 \%)$ & $4(30.5 \%)$ \\
\hline
\end{tabular}

Abbreviations: GMALL-German Multicenter Study Group for Adult ALL; CNS—central nervous system ETP—early-T precursor; WBC—white blood count; HGB - hemoglobin; PLT—platelets; CR—complete remission. $* n=45$ patients.

Overall, 20\% $(n=9)$ of patients had ETP-ALL/LBL immunophenotype, with CD5(-) (44.4\%) or CD5(+) ${ }^{\text {weaker }}$ (55.6\%) expression, co-expressed with CD34(100\%), HLA-DR(37.5\%), CD13(40\%), CD33(80\%), and CD15(25\%). According to the 2008 WHO subtyping, three patients (33\%) with ETP were categorized as ETP/pre-T/CD2(+) and six patients $(67 \%)$ as ETP/pro-T/CD2(-). There was no statistically significant difference in OS ( $p=0.135)$ among patients with the ETP vs. non-ETP subtype, with a five-year OS of $33 \%$ and $66 \%$, respectively, and a five-year EFS of $26 \%$ and $59 \%$, respectively $(p=0.095)$ (Figure 5). 


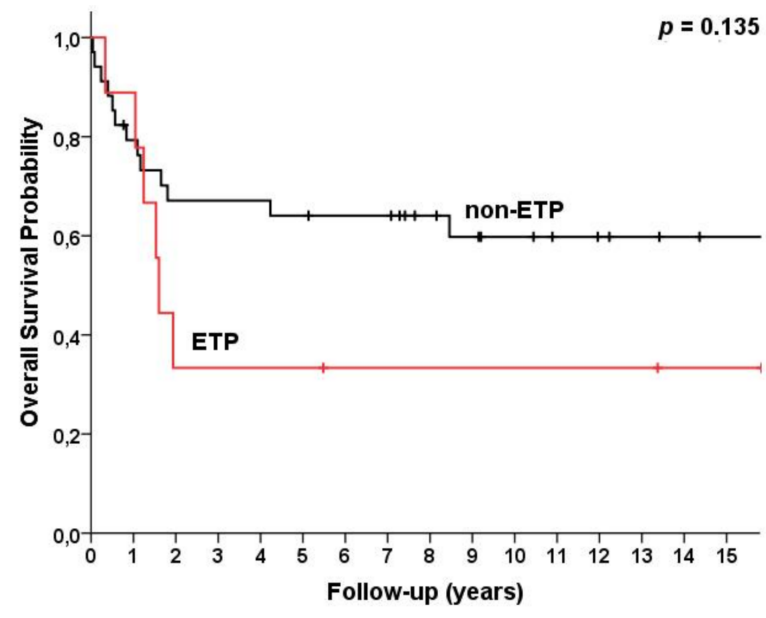

(a)

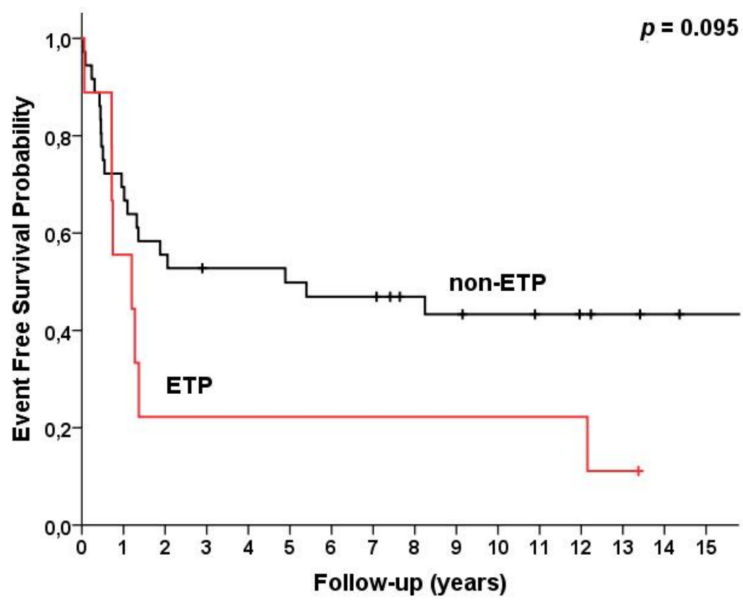

(b)

Figure 5. Survival for T-LBL $(n=45)$ patients categorized by subgroup defined by WHO 2017 as ETP $(n=9)$ vs. non-ETP $(n=36)$. (a) Overall survival and (b) event-free survival.

Among nine ETP patients, three $(33 \%)$ are alive to date. They include two out of the three patients with ETP/pre-T/CD2(+) and only one of the six patients with the ETP/proT/CD2(-) phenotype. Overall, $44.4 \%(n=4)$ of the ETP patients underwent SCT, two in the first line treatment (CR1), one in the second remission (CR2), and one in partial remission $(\mathrm{PR} /(\mathrm{MRD}+)$ after $\mathrm{CR} 1$ failure. Two of the three patients who are alive received SCT.

\section{Discussion}

T-LBL is a very rare disease with only few publications on its clinical characteristics and treatment outcomes. In contrast to leukemia (T-ALL), for T-LBL no risk model stratification has yet been defined. No clinical features or laboratory markers have been widely accepted as prognostic factors, except patients' age, usually defined as under or over 40 years; its prognostic significance is attributed to a lower tolerance of intensive chemotherapy in older patients. A prognostic model based on the rearrangements of four-genes, NOTCH1/FBXW7/RAS/PTEN, is important, but still difficult to apply in daily practice $[4,8]$. The role of immunophenotyping to determine blast maturity has also been recognized by the WHO 2008 classification, which distinguished four T-ALL/LBL ontogenetic subtypes. The WHO 2017 revision included a new provisional entity, an ETP subtype associated with very early ontogenesis $[1,2]$. Our study describes the immunophenotype distribution of T-LBL, classified according to the WHO 2008/2017 definitions, and the prognostic significance of an immunophenotype in the context of OS and EFS. We also analyzed the clinical features and outcomes in several T-LBL cases subjected to SCT. The clinical features of our patients did not differ from those described in most publications [5-7]. Primary CNS involvement was found in only three patients (6\%) and had no prognostic significance for treatment outcomes. Bone marrow involvement, based on HP assessment of trephine bone marrow biopsy specimens, was found in $22 \%$ of patients. Evaluation of bone marrow by FCM identified minimal BM involvement undetectable by trephine biopsy in nearly half of the cases. This indicates that flow cytometric examination is a more sensitive method for MRD detection in T-LBL. However, the role of MRD for T-LBL is uncertain [7]. In this study, all patients with minimal disseminated disease (MDD) obtained $\mathrm{BM}$ eradication, and BM involvement, as shown by uni/multivariate analyses, proved not to influence the outcomes. Only the age of $>35$ years was a powerful independent prognostic factor, influencing OS, EFS, and the risk of CR failure. Recently, we have demonstrated that in different types of aggressive lymphomas, FNAB/FCM presents a better diagnostic accuracy and effectiveness than the routine HP/IHC examination [20,21]. Therefore, at our institution, in all cases clinically suspected of T-ALL/LBL or with HP/IHC-confirmed 
T-ALL/LBL, we always aim to perform FNAB/FCM. In contrast to T-ALL, few data are available on the incidence or prognostic value of immunologic subtypes of adult T-LBL. Here, we report a series of consecutive 49 adult patients with T-LBL, and in 47 of these cases a comprehensive FCM analysis was performed. To the best of our knowledge, this is one of the largest published cohorts of T-LBL cases, including ETP-LBL cases (20\%) diagnosed by the FNAB/FCM.

The cortical subtype was found to be the most common, followed by the pro-T/CD2(-), pre-T/CD2(+), and medullary subtypes. In the largest group of 105 prospectively collected adult patients with T-LBL (GMALL) [9], the incidence of cortical and early or mature phenotypes was similar to those in our study. Contrary to this, according to a recent publication from China, among 64 T-LBL patients with FCM results, only $8 \%$ was of the cortical subtype, and the most frequent were the early and mature/medullary subtypes (70\% and $22 \%$, respectively) [15]. In our previous study, we showed no differences in the subtype frequency between LBL vs. ALL presentation [22,23]. In accordance with our data, a correlation between the stages of T-cell differentiation and survival, mainly in T-ALL, has been shown. Based on the results of GMALL 05/93 study, early and mature T-ALL were recognized as high-risk subtypes, and allo-SCT was recommended in CR1 [14,24]. In contrast to GMALL, in MD Anderson Cancer Center (MDACC) protocols the routine use of allo-SCT in CR1 was not recommended based on immunological subtypes regarded as high-risk by GMALL. Jain et al. [13] found no difference in CR rates, OS, and EFS, between the early, cortical, and mature subtypes. However, their subtyping was based mainly on $\mathrm{CD} 1 \mathrm{a}$ and sCD3 status, and the distinction between pro-T/CD2(-) and pre-T/CD2(+) has not been performed in most cases, so the proportion between the early/pro-T and early/pre-T subtypes was biased. In addition, the cortical subtype was recognized in only $24.5 \%$ of patients. Interestingly, the median OS of early/pre-T (CD2+) subtype patients was not reached. As recently presented by the Spanish PETHEMA group, the most favorable outcome in a T-ALL cohort was in the pre-T and cortical subtypes, with a four-year OS of $61 \%$ and $54 \%$, respectively, while it was only $30 \%$ and $39 \%$ in the ETP and mature subtypes, respectively [18]. Unfortunately, data on the outcomes of T-LBL patients are limited. In the above-mentioned studies, the GMALL LBL cohort presented a five-year OS slightly superior for the cortical (78\%) than for the early/mature subtype (58\%), while the China LBL cohort showed no difference in progression-free survival (PFS) between the immunophenotype subgroups $[9,15]$. In our previous studies, the most favorable outcomes were related to the cortical and early/pre-T/CD2(+) T-ALL/LBL subtypes, with a five-year OS of $69 \%$ and $48 \%$, respectively [22,23]. Considering the prognostic significance of CD2 expression, it is apparent that pro-T/CD2(-) and pre-T/CD2(+) cases should not be classified together as the early subtype. In our series of 47 T-LBL patients with comprehensive immunophenotyping, OS and EFS depended to a large extent on the 2008 WHO subtype. As expected, for the cortical (CD1a+) subtype, the 5/10-year OS and EFS were significantly better than those of the non-cortical subtypes. However, extremely poor outcomes were shown for the early pro-T/CD2(-) subtype as compared with all the other, non-pro-T subtypes. In the Cancer and Leukemia Group B (CALGB) series of patients treated according to the 8364 protocol, a number of T cell markers expressed in T-ALL cases was shown to be prognostically significant. In particular, patients expressing six or seven T cell antigens had a longer PFS and OS than patients expressing three or fewer antigens. In addition, patients with CD1a, CD2, CD4, and CD5 expression had significantly improved survival rates [25]. In our series, a five-year OS for patients with CD2, CD1a, and more than three pan-T Ags expressed was significantly higher than in patients with the lack of CD2 and CD1a and no more than three pan-T Ag expression. By multivariate analysis that incorporated the whole panel of antigens and numerous clinical data, negative $\mathrm{CD} 2$ status and age $>35$ years were the only and powerful independent prognostic factors influencing OS and EFS. To the best of our knowledge, the expression of CD2 in patients with T-ALL/LBL has not been clearly demonstrated as a prognostic factor. In the UKALLXII/ECOG 2993 study, an association between CD2 positivity and simple karyotypes was found in ALL/LBL. Patients expressing 
CD2 rarely had a complex karyotype (only $2 \%$ ), while $27 \%$ of the CD2(-) patients did have a complex karyotype. At the same time, a complex karyotype vs. a simple/normal karyotype was significantly associated with a lower five-year OS [12]. Our data are in line with the UKALLXII/ECOG 2993 study and suggest that a complex karyotype is more frequent in CD2-negative cases. However, the number of our cases with a karyotype tested was too small to prove statistical significance. Other studies have shown that $C D 2$ negativity correlated with the immature T-cell receptor and TCR $\gamma \delta$ T-ALL lineages, which are associated with the occurrence of the CALM-AF10 fusion gene and the TLX3/HOX11L2 and MLL(KMT2A) gene rearrangements, considered to be prognostically unfavorable [26-28].

CD2 is a cell adhesion molecule found on the surface of T cells and natural killer (NK) cells. In humans, it interacts with other adhesion molecules, such as lymphocyte function-associated antigen-3 (LFA-3/CD58), which are expressed on the surface of other cells. In addition to its adhesive properties, CD2 also acts as a co-stimulatory molecule for $\mathrm{T}$ and NK cells $[29,30]$. One may speculate that the lack of CD2 expression on TALL/LBL cells minimizes interactions between neoplastic and immune cells, enabling relapse even from the MRD level. In our series, $27.7 \%$ of T-LBL was CD2(-). Although there was no major difference in the baseline characteristics between the CD2(-) and CD2(+) T-LBL patients, the CD2(-) patients showed a higher percentage of induction failure than the CD2(+) patients, and only $69 \%$ achieved CR, compared with $91 \%$ in the CD2(+) patients. Progression occurred in up to $77 \%$ of the CD2(-) patients, compared to only $20.5 \%$ for CD2(+). Only four $(30.7 \%)$ of the CD2(-) patients are alive; all received allo-SCT. The majority (69\%) of CD2(-) patients had the pro-T subtype, but the loss of $\mathrm{CD} 2$ expression is also rare in the other subtypes. In two cases, the lack of CD2 was identified in the cortical phenotype associated with an overall good prognosis, but both patients experienced recurrence of the disease in the CNS. One had a confirmed lymphoma infiltration in the spinal canal, the other had a minimal CSF involvement by FCM. Both patients have remained in CR2 after allo-SCT (for 11 and 3 years until now). Six out of thirteen $(46 \%)$ of our CD2(-) patients met the criteria of the ETP subgroup.

Many cases previously classified as pro-T or pre-T would now meet the criteria for ETP. Among immunological subtypes, ETP is now the most widely discussed, but there is still controversy over the prognostic significance of the ETP T-ALL/LBL subgroup. In the MDACC series of T-ALL/LBL patients, $17 \%$ were recognized as ETP, and of the ETP patients for whom sufficient data were available, approximately half were categorized as early/pro-T and half as early/pre-T subtypes; only four patients with ETP had LBL presentation. ETP patients had significantly worse OS than patients with non-ETP ALL, with no difference in EFS [13]. Recently, in the Group for Research on Adult Acute Lymphoblastic Leukemia (GRAALL) 2003 and 2005 studies, 22.1\% of patients with the ETP phenotype were treated with the pediatric-type regimen. Based on this intensive strategy, the overall prognosis of ETP-ALL was similar to that of the rest of the T-ALL cohort, with the impressive five-year OS of $59.6 \%$ and $66.5 \%$ for ETP and non-ETP, respectively [17]. An important difference was observed in the rate of SCT for patients in the first CR between the MDACC and GRAALL studies, with only $17.6 \%$ patients transplanted in MDACC and $48.9 \%$ in GRAALL. It was concluded by GRAALL that the ETP subgroup is a specific predictor of benefiting from allo-SCT [17]. On the other hand, the above-mentioned retrospective analysis by PETHEMA included 34 patients with ETP-ALL (20\% of T-ALL cases) and revealed that treatment intensification with allo-SCT did not improve survival (with a five-year OS of 36\%) [18]. Data from the Chinese study referred to above suggest that autologous SCT might overcome the unfavorable effect of the ETP-LBL subtype on prognosis [15]. In our series, $20 \%$ of the T-LBL patients were identified with ETP, a similar frequency to that found in the MDACC, PETHEMA, and GRAALL studies, but other than in the MDACC cohort, a higher percentage of our patients were categorized as pro-T-CD2(-)/ETP $(66.6 \%)$ than as pre-T-CD2(+)/ETP (33.4\%) [13]. In contrast to the CD2(-) patients as a whole subgroup, the ETP patients had a low risk of induction failure, with $88.8 \%$ of $C R$, while progression was twice as common in ETP $(66.6 \%)$ compared to non-ETP (30.5\%) patients. The ETP 
phenotype was not shown to influence OS and EFS, unlike the CD2(-) pro-T subtype. Among nine ETP patients, three are alive. They include two out of the three patients with ETP/pre-T/CD2(+) and only one of the six patients with the ETP/pro-T/CD2(-) phenotype. One could speculate that patients with ETP/pre-T/CD2(+) may have a better prognosis. In our small group of patients who had received allo-SCT, $50 \%(2 / 4)$ of the ETP patients and $80 \%(4 / 5)$ of the CD2(-) patients benefited from this procedure, but the optimal management remains unclear. It is likely that more targeted treatments are needed to improve the remission rate for the early T-ALL/LBL classified as ETP, and in our opinion also defined as CD2(-).

\section{Conclusions}

Our data show that (1) CD2 status, along with an age of less or over 35 years, is a powerful independent prognostic factor influencing OS and the risk of treatment failure; (2) the early/pro-T/CD2(-) subtype is associated with extremely poor outcomes, while all the other, non-pro-T subtypes show significantly better outcomes; (3) OS and EFS strongly relate to the 2008 WHO subtypes; (4) the cortical subtype is associated with a significantly better five-year OS and EFS than the non-cortical subtype; (5) poor outcomes in ETP vs. non-ETP are strikingly consistent with the pro-T/CD2(-) subtype.

To conclude, the lack of CD2 expression in T-LBL emerges as a new marker of an ultra-high-risk of treatment failure. This context casts doubts on the concept of ETP as a category of high-risk disease in all cases because, as we show here, ETP is a non-uniform entity, where the outcome depends on the CD2 status.

Supplementary Materials: The following are available online at https://www.mdpi.com/article/ 10.3390/cancers13081911/s1, Table S1: (A) A minimal panel of anti-human monoclonal antibodies (mAbs) used for the flow cytometry (FCM) analysis, (B) Additional mAbs used for extended FCM diagnostics; Table S2: Details of GMALL 05/93 and GMALL T-LBL 1/2004 chemotherapy; Table S3: Baseline diagnostic characteristics of T-LBL patients; Table S4: Clinical and immunophenotype characteristics, and outcome for T-LBL CD2(-) patients; Table S5: Immunophenotype details (WHO 2008 T-ALL/LBL subtypes).

Author Contributions: Conceptualization, B.O., G.R. and J.W.; methodology, B.O., G.R.; validation, G.R., K.B., Z.B., A.B.; formal analysis, W.M., S.R.; investigation, B.O., G.R., M.Ch., K.B., K.D.-C., Z.B., J.R.-J., A.B., S.R., W.M.; resources, K.D.-C., J.R.-J., B.O., J.W.; writing—original draft preparation, B.O., G.R. and M.Ch.; writing—review and editing, B.O., G.R., M.Ch. and J.W.; visualization, W.M., S.R., B.O., M.Ch., G.R.; supervision, B.O. All authors have read and agreed to the published version of the manuscript.

Funding: This research received no external funding.

Institutional Review Board Statement: Ethical review and approval were waived for this study, as it comprises retrospective analyses of treatment results and clinico-pathological characteristics of patients.

Informed Consent Statement: Not applicable.

Data Availability Statement: The data presented in this study are available in this article (and supplementary material).

Acknowledgments: The authors wish to thank Elzbieta Kulczycka, for performing the immunohistochemical staining, Maciej Turski for participating in the CT-guided FNAB, and Michel Buffet, for the critical reading of the manuscript.

Conflicts of Interest: J.W.-Advisory role: Roche, Celgene, Takeda, Janssen-Cilag, Servier, Amgen, BMS, Gielad, Novartis, Abbvie, research funding: Roche, GSK/Novartis; lecture honoraria: Roche, Celgene, Takeda, Janssen-Cilag, Servier, Gielad, Abbvie; travel support: Roche. The other authors declare no conflict of interest. 


\section{References}

1. Borowitz, M.J.; Chan, J.K.C. T lymphoblastic leukaemia/lymphoma. In WHO Classification of Tumours of Haematopoietic and Lymphoid Tissues, 4th ed.; Swerdlow, S.H., Campo, E., Harris, N.L., Eds.; IARC: Lyon, France, 2008; pp. 176-178.

2. Borowitz, M.J.; Chan, J.K.C. T lymphoblastic leukaemia/lymphoma. In WHO Classification of Tumours of Haematopoietic and Lymphoid Tissues, Revised, 4th ed.; Swerdlow, S.H., Campo, E., Harris, N.L., Eds.; IARC: Lyon, France, 2017; pp. $209-212$.

3. Le Gouill, S.; Lepretre, S.; Briere, J.; Morel, P.; Bouabdallah, R.; Raffoux, E.; Sebban, C.; Lepage, E.; Brice, P. Adult lymphoblastic lymphoma: A retrospective analysis of 92 patients under 61 years included in the LNH87/93 trials. Leukemia 2003, 17, 2220-2224. [CrossRef] [PubMed]

4. Lepretre, S.; Graux, C.; Touzart, A.; Macintyre, E.; Boissel, N. Adult T-type lymphoblastic lymphoma: Treatment advances and prognostic indicators. Exp. Hematol. 2017, 51, 7-16. [CrossRef] [PubMed]

5. Hoelzer, D.; Gokbuget, N.; Digel, W.; Faak, T.; Kneba, M.; Reutzel, R.; Romejko-Jarosinska, J.; Zwolinski, J.; Walewski, J. Outcome of adult patients with T-lymphoblastic lymphoma treated according to protocols for acute lymphoblastic leukemia. Blood 2002, 99, 4379-4385. [CrossRef] [PubMed]

6. Thomas, D.A.; O’Brien, S.; Cortes, J.; Giles, F.J.; Faderl, S.; Verstovsek, S.; Ferrajoli, A.; Koller, C.; Beran, M.; Pierce, S.; et al. Outcome with the hyper-CVAD regimens in lymphoblastic lymphoma. Blood 2004, 104, 1624-1630. [CrossRef]

7. Cortelazzo, S.; Intermesoli, T.; Oldani, E.; Ciceri, F.; Rossi, G.; Pogliani, E.M.; Mattei, D.; Romani, C.; Cortelezzi, A.; Borlenghi, E.; et al. Results of a lymphoblastic leukemia-like chemotherapy program with risk-adapted mediastinal irradiation and stem cell transplantation for adult patients with lymphoblastic lymphoma. Ann. Hematol. 2012, 91, 73-82. [CrossRef]

8. Lepretre, S.; Touzart, A.; Vermeulin, T.; Picquenot, J.M.; Tanguy-Schmidt, A.; Salles, G.; Lamy, T.; Béné, M.-C.; Raffoux, E.; Huguet, F.; et al. Pediatric-Like Acute Lymphoblastic Leukemia Therapy in Adults With Lymphoblastic Lymphoma: The GRAALL-LYSA LL03 Study. J. Clin. Oncol. 2016, 34, 572-580. [CrossRef]

9. Gökbuget, N.; Wolf, A.; Stelljes, M.; Hüttmann, A.; Buss, E.C.; Viardot, A.; Brandt, K.; de Wit, M.; Frickhofen, N.; Kebenko, M.; et al. Favorable Outcome in a Large Cohort of Prospectively Treated Adult Patients with T-Lymphoblastic Lymphoma (T-LBL) Despite Slowly Evolving Complete Remission Assessed By Conventional Radiography. Blood 2014, 124, 370. [CrossRef]

10. El-Fattah, M.A. Prognostic Factors and Outcomes of Adult Lymphoblastic Lymphoma in the United States. Clin. Lymphoma Myeloma Leuk. 2017, 17, 498-505.e6. [CrossRef]

11. Ellin, F.; Jerkeman, M.; Hagberg, H.; Relander, T. Treatment outcome in T-cell lymphoblastic lymphoma in adults-A populationbased study from the Swedish Lymphoma Registry. Acta Oncol. 2014, 53, 927-934. [CrossRef]

12. Marks, D.I.; Paietta, E.M.; Moorman, A.V.; Richards, S.M.; Buck, G.; DeWald, G.; Ferrando, A.; Fielding, A.K.; Goldstone, A.H.; Ketterling, R.P.; et al. T-cell acute lymphoblastic leukemia in adults: Clinical features, immunophenotype, cytogenetics, and outcome from the large randomized prospective trial (UKALL XII/ECOG 2993). Blood 2009, 114, 5136-5145. [CrossRef]

13. Jain, N.; Lamb, A.V.; O’Brien, S.; Ravandi, F.; Konopleva, M.; Jabbour, E.; Zuo, Z.; Jorgensen, J.; Lin, P.; Pierce, S.; et al. Early T-cell precursor acute lymphoblastic leukemia/lymphoma (ETP-ALL/LBL) in adolescents and adults: A high-risk subtype. Blood 2016, 127, 1863-1869. [CrossRef]

14. Hoelzer, D.; Thiel, E.; Arnold, R.; Beck, J.; Beelen, D.W.; Bornhäuser, M.; Bunjes, D.; Ditz, D.; Dührsen, U.; Finke, J.; et al. Successful Subtype Oriented Treatment Strategies in Adult T-All; Results of 744 Patients Treated in Three Consecutive GMALL Studies. Blood 2009, 114, 324. [CrossRef]

15. Liu, Y.; Rao, J.; Li, J.; Wen, Q.; Wang, S.; Lou, S.; Yang, T.; Li, B.; Gao, L.; Zhanget, C.; et al. Tandem autologous hematopoietic stem cell transplantation for treatment of adult T-cell lymphoblastic lymphoma: A multiple center prospective study in China. Haematologica 2021, 106, 163-172. [CrossRef]

16. Coustan-Smith, E.; Mullighan, C.G.; Onciu, M.; Behm, F.G.; Raimondi, S.C.; Pei, D.; Cheng, C.; Su, X.; Rubnitz, J.E.; Basso, G.; et al. Early T-cell precursor leukaemia: A subtype of very high-risk acute lymphoblastic leukaemia. Lancet Oncol. 2009, 10, 147-156. [CrossRef]

17. Bond, J.; Graux, C.; Lhermitte, L.; Lara, D.; Cluzeau, T.; Leguay, T.; Cieslak, A.; Trinquand, A.; Pastoret, C.; Belhocine, M.; et al. Early Response-Based Therapy Stratification Improves Survival in Adult Early Thymic Precursor Acute Lymphoblastic Leukemia: A Group for Research on Adult Acute Lymphoblastic Leukemia Study. J. Clin. Oncol. 2017, 35, 2683-2691. [CrossRef]

18. Genesca, E.; Morgades, M.; Montesinos, P.; Barba, P.; Gil, C.; Guardia, R.; Moreno, M.-J.; Martínez-Carballeira, D.; García-Cadenas, I.; Vives, S.; et al. Unique clinico-biological, genetic and prognostic features of adult early T-cell precursor acute lymphoblastic leukemia. Haematologica 2020, 105, e294-e297. [CrossRef]

19. Castaneda Puglianini, O.; Papadantonakis, N. Early precursor T-cell acute lymphoblastic leukemia: Current paradigms and evolving concepts. Ther. Adv. Hematol. 2020, 11, 2040620720929475. [CrossRef]

20. Rymkiewicz, G.; Grygalewicz, B.; Chechlinska, M.; Blachnio, K.; Bystydzienski, Z.; Romejko-Jarosinska, J.; Woroniecka, R.; Zajdel, M.; Domanska-Czyz, K.; Martin-Garcia, D.; et al. A comprehensive flow-cytometry-based immunophenotypic characterization of Burkitt-like lymphoma with 11q aberration. Mod. Pathol. 2018, 31, 732-743. [CrossRef]

21. Woroniecka, R.; Rymkiewicz, G.; Grygalewicz, B.; Blachnio, K.; Rygier, J.; Jarmuz-Szymczak, M.; Ratajczak, B.; Pieńkowska-Grela, B. Cytogenetic and flow cytometry evaluation of Richter syndrome reveals MYC, CDKN2A, IGH alterations with loss of CD52, CD62L and increase of CD71 antigen expression as the most frequent recurrent abnormalities. Am. J. Clin. Pathol. 2015, 143, 25-35. [CrossRef] 
22. Ostrowska, B.; Rymkiewicz, G.; Domanska-Czyz, K.; Romejko-Jarosińska, J.; Błachnio, K.; Popławska, L.; Michalski, W.; Walewski, J. Pro-T cell ALL/LBL: An ultra-high risk CD2-negative disease subtype in adults defined by flow cytometry. Haematologica 2017, $102,30-31$

23. Ostrowska, B.; Rymkiewicz, G.; Romejko-Jarosinska, J.; Blachnio, K.; Domanska-Czyz, K.; Osiadacz, W.; Osowiecki, M.; Paszkiewicz-Kozik, E.; Poplawska, L.; Rozewska, D.; et al. Fine Needle Aspiration Biopsy with Flow Cytometry is a Reliable Method for Defining Immunophenotype of Prognostic Value in T Lymphoblastic Lymphoma. Blood 2009, 114, 3928. [CrossRef]

24. Gökbuget, N.; Arnold, R.; Böhme, A.; Fietkau, R.; Freund, M.; Ganser, A.; Kneba, M.; Lipp, T.; Ludwig, W.-D.; Maschmeyer, G.; et al. Treatment of Adult ALL According to Protocols of the German Multicenter Study Group for Adult ALL (GMALL). In Acute Leukemias; Springer: Berlin/Heidelberg, Germany, 2008; pp. 167-176.

25. Czuczman, M.S.; Dodge, R.K.; Stewart, C.C.; Frankel, S.R.; Davey, F.R.; Powell, B.L.; Szatrowski, T.P.; Schiffer, C.A.; Larson, R.A.; Bloomfield, C.D. Value of immunophenotype in intensively treated adult acute lymphoblastic leukemia: Cancer and leukemia Group B study 8364. Blood 1999, 93, 3931-3939. [PubMed]

26. Asnafi, V.; Radford-Weiss, I.; Dastugue, N.; Bayle, C.; Leboeuf, D.; Charrin, C.; Garand, R.; Lafage-Pochitaloff, M.; Delabesse, E.; Buzyn, A.; et al. CALM-AF10 is a common fusion transcript in T-ALL and is specific to the TCRgammadelta lineage. Blood 2003, 102, 1000-1006. [CrossRef] [PubMed]

27. Asnafi, V.; Buzyn, A.; Thomas, X.; Huguet, F.; Vey, N.; Boiron, J.M.; Reman, O.; Cayuela, J.-M.; Lheritier, V.; Vernant, J.-P.; et al. Impact of TCR status and genotype on outcome in adult T-cell acute lymphoblastic leukemia: A LALA-94 study. Blood 2005, 105, 3072-3078. [CrossRef]

28. Baleydier, F.; Decouvelaere, A.V.; Bergeron, J.; Gaulard, P.; Canioni, D.; Bertrand, Y.; Lepretre, S.; Petit, B.; Dombret, H.; Beldjord, K.; et al. T cell receptor genotyping and HOXA/TLX1 expression define three T lymphoblastic lymphoma subsets which might affect clinical outcome. Clin. Cancer Res. 2008, 14, 692-700. [CrossRef]

29. Moingeon, P.; Chang, H.C.; Wallner, B.P.; Stebbins, C.; Frey, A.Z.; Reinherz, E.L. CD2-mediated adhesion facilitates T lymphocyte antigen recognition function. Nature 1989, 339, 312-314. [CrossRef]

30. Crawford, K.; Stark, A.; Kitchens, B.; Sternheim, K.; Pantazopoulos, V.; Triantafellow, E.; Wang, Z.; Vasir, B.; Larsen, C.E.; Gabuzdaet, D.; et al. CD2 engagement induces dendritic cell activation: Implications for immune surveillance and T-cell activation. Blood 2003, 102, 1745-1752. [CrossRef] 